\title{
IMAGE-BASED VEHICLE TRACKING FROM ROADSIDE LIDAR DATA
}

\author{
J. Zhang ${ }^{1}$, W. Xiao ${ }^{1}$, B. Coifman ${ }^{2}$, J. P. Mills ${ }^{1}$ \\ ${ }^{1}$ School of Engineering, Newcastle University, Newcastle upon Tyne, UK - (j.zhang85, wen.xiao, jon.mills)@ newcastle.ac.uk \\ ${ }^{2}$ Department of Civil, Environmental and Geodetic Engineering / Department of Electrical and Computer Engineering, \\ The Ohio State University, Columbus, USA - coifman.1@ osu.edu
}

\author{
Commission II, WG II/3
}

KEY WORDS: Image registration, lidar, template matching, vehicle detection, vehicle tracking

\begin{abstract}
:
Vehicle tracking is of great importance in urban traffic systems, and the adoption of lidar technologies - including on-board and roadside systems - has significant potential for such applications. This research therefore proposes and develops an image-based vehicle-tracking framework from roadside lidar data to track the precise location and speed of a vehicle. Prior to tracking, vehicles are detected in point clouds through a three-step procedure. Cluster tracking then provides initial tracking results. The second tracking stage aims to provide more precise results, in which two strategies are developed and tested: frame-by-frame and modelmatching strategies. For each strategy, tracking is implemented through two threads by converting the 3D point cloud clusters into 2D images relating to the plan and side views along the tracked vehicle's trajectory. During this process, image registration is exploited in order to retrieve the transformation parameters between every image pair. Based on these transformations, vehicle speeds are determined directly based on (a) the locations of the chosen tracking point in the first strategy; (b) a vehicle model is built and tracking point locations can be calculated after matching every frame with the model in the second strategy. In contrast with other existing methods, the proposed method provides improved vehicle tracking via points instead of clusters. Moreover, tracking in a decomposed manner provides an opportunity to cross-validate the results from different views. The effectiveness of this method has been evaluated using roadside lidar data obtained by a Robosense 32-line laser scanner.
\end{abstract}

\section{INTRODUCTION}

Efficient vehicle tracking is a critical component of many Intelligent Transportation Systems (ITS) and is of great importance for the improvement of both traffic conditions and the environment. The ability to track vehicles through a controlled area can help observe, and hence prevent traffic violations such as speeding, frequent lane changes and drink driving (Sanchez et al., 2011). Furthermore, vehicle tracking provides an efficient means to study the interaction between vehicle dynamics and emissions (Khalfan et al., 2015).

Recently, interest in lidar technologies in the field of vehicle tracking has increased due to reduced cost and high fidelity of point cloud measurements (Shirazi and Morris, 2017). Moreover, mobile or fixed lidar systems usually consist of a certain number of laser arrays rotating rapidly around the vertical axis so that the surroundings are continuously scanned, greatly improving the completeness of the acquired vehicle data (Xiao et al., 2017). Another important fact is that lidar data contains 3D information of vehicles, which is essential for traffic modelling. Yao et al. (2012) investigated the theoretical background for airborne laser scanning (ALS) systems that were used to monitor traffic from airborne platforms. Although the results showed potential in supporting traffic monitoring applications, they were not comparable with those of optical or ground-based sensors. Luo et al. (2016) published a novel realtime multiple vehicle detection and tracking algorithm based purely on a Velodyne HDL-32E sensor that can be decomposed into three steps: segmentation, clustering and tracking. A fast and efficient real time clustering algorithm called the radially bounded nearest neighbour (RBNN) is used in the second step. A Hungarian algorithm procedure and adaptive Kalman filtering are used for data association and tracking. Chen et al. (2016) provided a dynamic vehicle detection and tracking algorithm to solve the problem that dynamic vehicles occluded by other objects cannot be detected and tracked by other methods. For tracking dynamic vehicles, the Scaling Series algorithm coupled with a Bayesian Filter (SSBF) is improved by adding the egomotion compensation so that it is able to update the pose and velocity for each vehicle in dynamic background scenes.

As opposed to lidar applications on autonomous vehicles, as described above, for roadside traffic surveillance lidar sensors are usually installed at a fixed location instead of on a moving vehicle. The vehicle tracking system should be scalable and robust to track vehicles with fewer lidar points. Sun et al. (2018) developed a framework specifically to extract high-resolution vehicle trajectories from roadside lidar sensors. The procedure involved three main steps: vehicle data cloud clustering; vehicle location estimation through a principle component-based oriented bounding box method; and geometrically-based tracking. Wu et al. (2018) presented an automatic procedure for vehicle tracking with a roadside lidar sensor involving background filtering, lane identification, and vehicle speed tracking. Notably, for vehicle tracking from roadside lidar data, a number of previous studies used the average of all points in a cluster as the tracking point, which may cause large errors as the location and number of points in one cluster can vary with distance from the lidar sensor. To continuously track the location and speed of a vehicle more precisely, the point nearest to the lidar sensor on the vehicle is selected as the tracking point (Sun et al., 2018; Wu et al., 2018). More specifically, when the target vehicle is approaching the lidar, the nearest point is the front corner point. When it is leaving the lidar, the nearest point is the back corner point of the vehicle. 


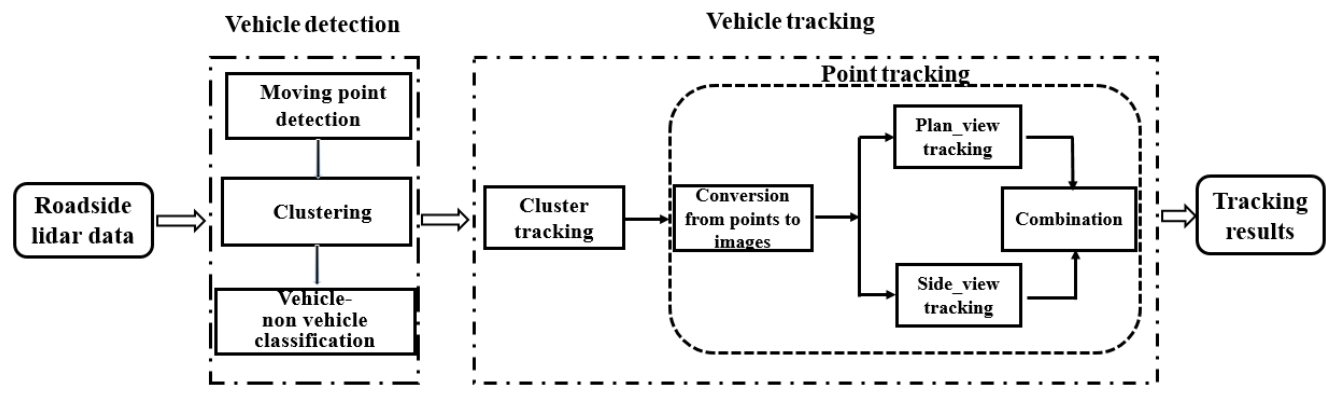

Figure 1. Framework of the proposed methodology.

Even though the adopted strategy provides improvements on previous work, it is still not as precise and convenient as choosing a fixed point throughout the entire tracking process. To locate a selected tracking point in each frame, it is necessary to determine the relationship between every two successive vehicle clusters via registration. Many studies have employed variants of the Iterative Closest Point (ICP) algorithm (Besl and McKay, 1992) for local registration of 3D overlapping scans (e.g. Byun et al., 2017). The algorithm iteratively establishes correspondences between the points of two point cloud datasets, computes the spatial distances between them and terminates when the sum of the spatial distances between the correspondences is at a minimum (Sanchez et al., 2017). However, this approach is computationally expensive since it requires an extensive search of point correspondences between the point clouds (Godin et al., 1994). Moreover, this method faces local minima issues and often needs a coarse initial alignment to converge to the optimum solution (Sanchez et al., 2017). To avoid such problems in 3D point cloud registration, 2D image-based point cloud registration approaches have been proposed in some studies (e.g. Lin et al., 2017; Christodoulou, 2018).

In order to perform vehicle tracking from roadside lidar data continuously and precisely, this study focuses on an imagebased tracking framework via a fixed point. By converting each vehicle 3D lidar point cluster to a $2 \mathrm{D}$ image in both the plan and side view along the vehicle trajectory, the $3 \mathrm{D}$ point cloudbased vehicle tracking problem is decomposed into two imagebased vehicle tracking procedures. To locate the selected tracking point in each frame, image registration is performed using template matching to determine the transformation parameters for every two successive images. The proposed method aims to provide improved vehicle tracking via points instead of clusters. In addition, tracking in a decomposed manner provides an opportunity to cross-validate the results from different views.

\section{METHODOLOGY}

A tracking-by-detection strategy (Asvadi et al., 2016) is adopted in the proposed method consisting of two components: vehicle detection and vehicle tracking, as illustrated in Figure 1.

\subsection{Vehicle detection}

The vehicle detection stage comprises three sub-steps: moving point detection; clustering; vehicle and non-vehicle classification. The Max-Distance method is adopted for moving point detection, after which the extracted moving points (including those on vehicles and non-vehicles) are grouped through a simple Euclidean Cluster Extraction algorithm. A traditional but efficient machine learning strategy, the Support Vector Machine (SVM), is used for vehicle and non-vehicle classification, and 3D object level features are selected to distinguish two classes of clusters.

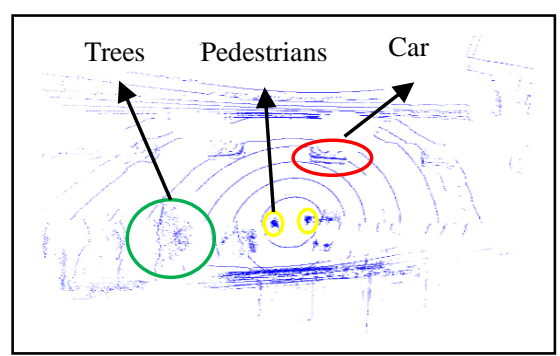

(a)

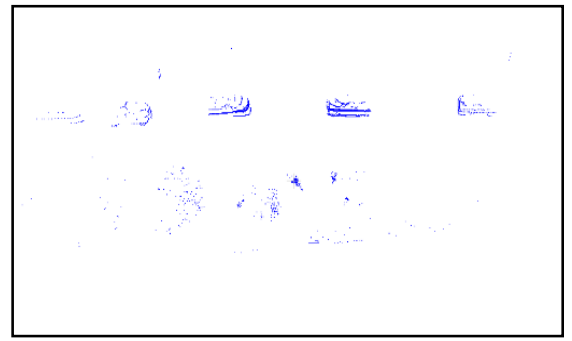

(b)

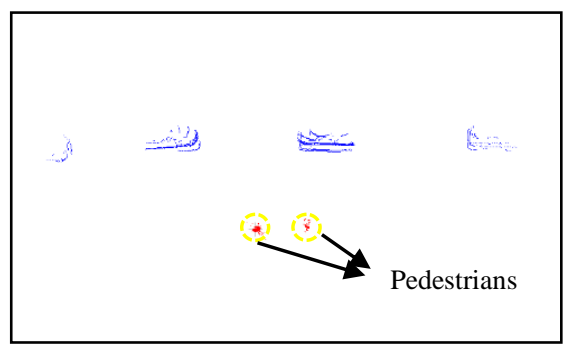

(c)

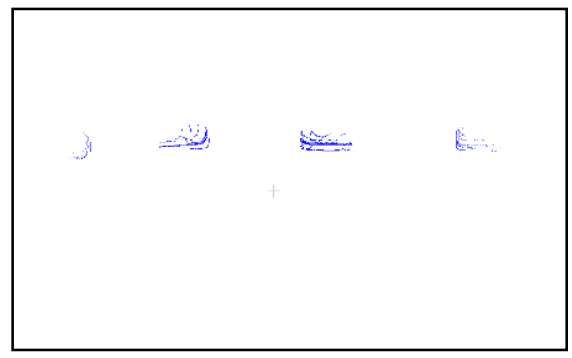

(d)

Figure 2. (a) Original frame (b) moving points (c) clustering results (d) classification results.

2.1.1 Moving point detection: The Max-Distance method (Xiao et al., 2016a) is utilized for moving point detection from 
static laser scanning. The principle is straightforward: The static environment is assumed impenetrable and only the furthest points of each laser beam are considered to be located on the static background. Therefore, points on moving objects appear between the laser scanner centre and the far end points and they can be extracted by constructing the background (Figure 2(b)).

2.1.2 Clustering: The Euclidean Cluster Extraction algorithm is used in this step. Here, two parameters are important: the cluster size $S$, and the cluster tolerance $d$. The minimum cluster size $S_{1}$ and the maximum cluster size $S_{2}$ should be determined according to the dataset. For cluster tolerance $d$, if the value is too small, an actual object can be seen as multiple clusters. Conversely, if the value is too large, multiple objects will be regarded as one cluster. Therefore, heuristic testing is required to determine the optimal value for the dataset. Figure 2(c) shows the clustering result for the extracted moving points.

2.1.3 Vehicle and non-vehicle classification: Traditional machine learning is an efficient method for vehicle detection from lidar data, in which two important aspects are feature extraction and sample selection. Low-level features based on an individual point or a small group of points, such as dimensionality, are used in of the majority of studies (Demantké et al., 2011). In this case, the input for vehicle detection is moving point clusters, so the features extracted in the proposed method are at object level in 3D (Hwang et al., 2016; Xiao et al., 2016b):

(1) Standard deviations of coordinates $\mathrm{x}, \mathrm{y}, \mathrm{z}$ for points in the bounding box $(\operatorname{std}(x), \operatorname{std}(y), \operatorname{std}(z))$;

(2) The volume size of the bounding box: depth, width, max_height, min_height $\left(\mathrm{d}, \mathrm{w}, \mathrm{h}_{1}, \mathrm{~h}_{2}\right)$;

(3) Vertical point distribution histogram (v1 . . v10): if the input object is vertically divided into even parts from a certain height to the ground, a histogram containing the point proportion regarding the overall number of points at each vertical part can be regarded as the feature to distinguish vehicle and non-vehicles. The vehicles are divided into 10 parts and each is $20 \mathrm{~cm}$ high based on the assumption that maximum height of studied vehicles is lower than $2 \mathrm{~m}$.

Since this classification is dealt with as a binary problem, the training sample dataset mainly includes vehicles and nonvehicles (referring to all the other road users). These samples were chosen manually using CloudCompare software from the frames processed by the clustering operation. A SVM with RBF kernel was used as the classifier.

\subsection{Vehicle tracking}

To obtain improved precision, the proposed framework is composed of two parts: cluster tracking and point tracking. The first component is to provide initial results by finding the clusters for the target vehicle in the frames. Subsequently, the second component focuses on locating the chosen tracking point in every frame to obtain refined results.

2.2.1 Cluster tracking: Cluster tracking refers to identifying clusters belonging to the same car in each successive frame. The principles to judge whether two clusters in consecutive frames belong to the same car are described as:
(1) The similarity degree between two feature matrices is larger than $t_{1}$, here, the features are the same as those used in vehicle detection;

(2) The physical distance is smaller than $t_{2}$;

(3) The orientation change between two clusters is within $\left(-t_{3}, t_{3}\right)$. It is can be determined according to practice: firstly, as the frequency of the roadside scanner is extremely high and the speed of vehicles on city roads is limited, a judgement can be made that the geometrical similarity of two successive clusters belonging to the same car is much higher than that of different cars. Therefore, $t_{1}$ is defined to be 0.9 . Secondly, $\mathrm{t}_{2}=\mathrm{V} * \mathrm{~T}$, here, $\mathrm{V}$ is the maximum speed in the study area complying with the road speed limitation regulations in United Kingdom. $\mathrm{T}$ is the time interval between two frames from the laser scanner. Finally, the orientation of the car does not change greatly during short time interval, even when the car is changing lanes or turning. Here, $t_{3}$ is set to 10 degrees. Combining $t_{2}$ and $t_{3}$, the search range is a sector with $\mathrm{R}=\mathrm{t}_{1}$ and $\theta=20$ degrees.

The cluster tracking algorithm is illustrated in Figure 3.

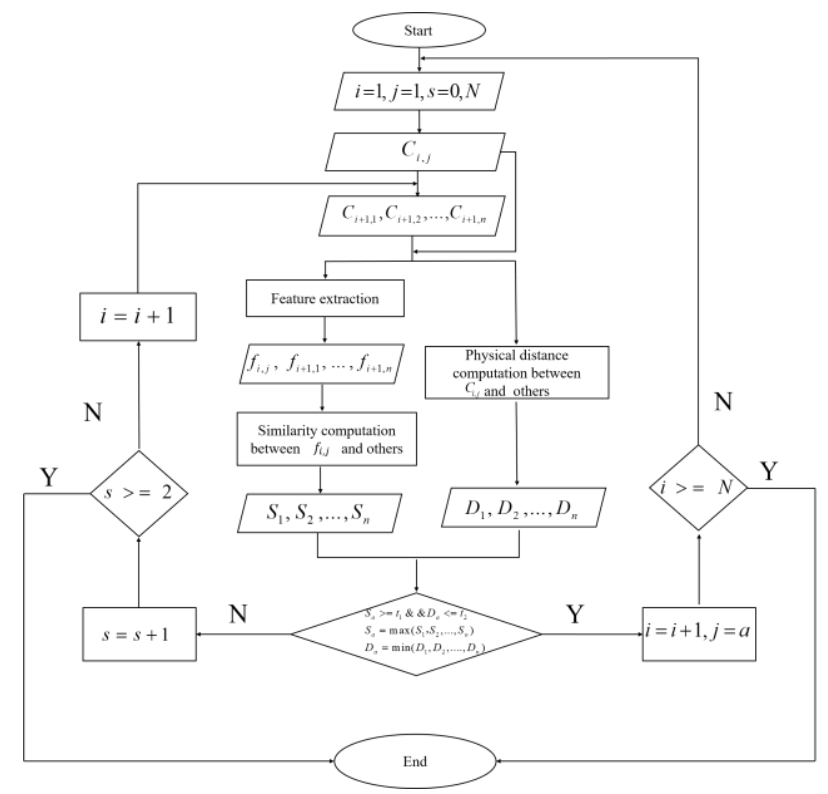

Figure 3. Cluster tracking algorithm.

2.2.2 Point tracking: In roadside laser scanning, vehicles on the road can only be partially scanned when they are passing through the laser scanner. The number of points in a vehicle cluster varies with the distance from the lidar sensor, and consequently the centre of the bounding box (usually regarded as the vehicle position) is also changing. This is illustrated in Figure 4 by the yellow line in the time-space diagram. Two parallel lines (red and blue lines in Figure 4) demonstrate the trends of the front and rear parts of the vehicle. It can be seen that the trend of the yellow line is different to the other two. However, if a fixed tracking point, for instance, the real centroid of the vehicle, is chosen during the process then the yellow line will be corrected to the green one.

Two strategies are presented to solve this issue. A frame-byframe strategy is used to locate the tracking point directly in two 
successive frames according to the transformation between them A second, model-based, strategy further builds a vehicle model based on the obtained transformations within every frame pair, then takes the determined centroid as the tracking point and back locates it in each frame via model fitting.

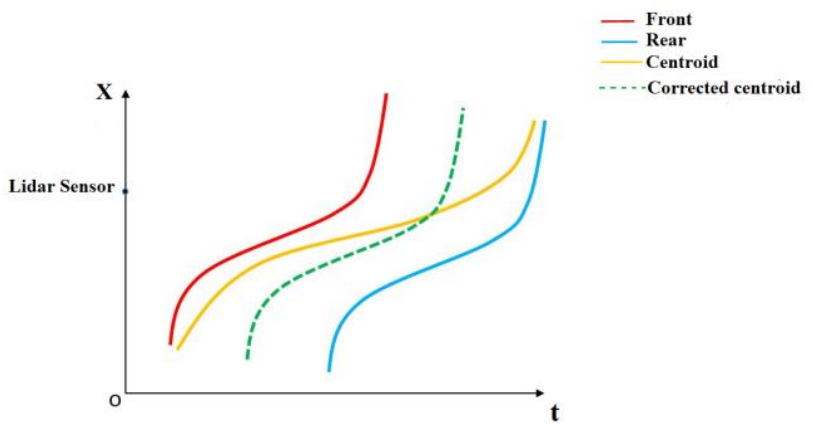

Figure 4. Time-space diagram of the target vehicle.

Instead of using 3D lidar points directly, image-based vehicle tracking is performed by converting 3D lidar point clusters into 2D images. Decomposing the 3D tracking problem into two 2D procedures on the plan and side views also means that the tracking results can be cross-validated. Since the tracking flows for the two threads are similar, the plan view tracking thread is taken here as an example to depict in detail, as shown in Figure 5 (for the model-based strategy).

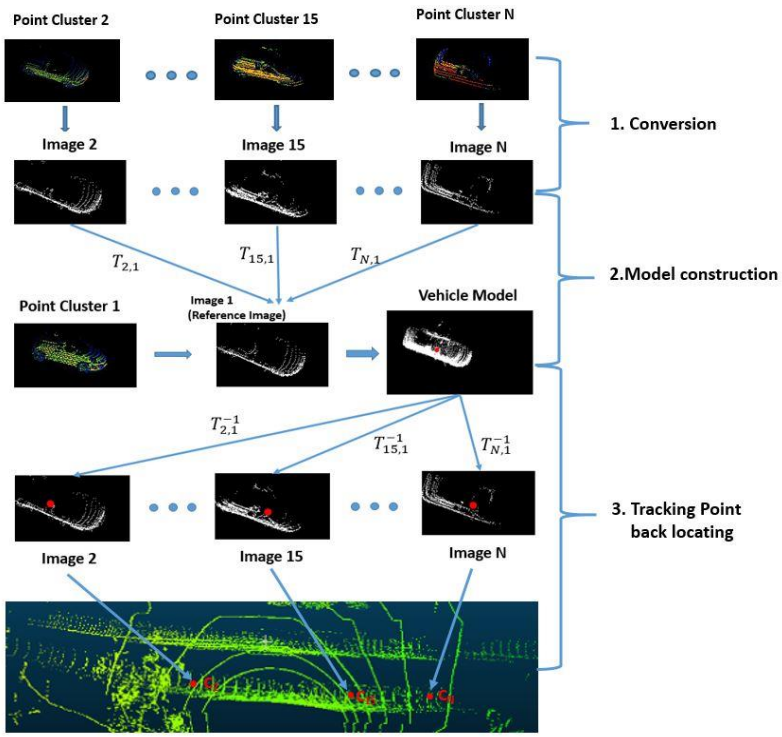

Figure 5. Plan view tracking.

Conversion: in the conversion step, each vehicle cluster composed of 3D lidar points is converted into a 2D planimetric image or side-view image, as shown in Figure 6.

W
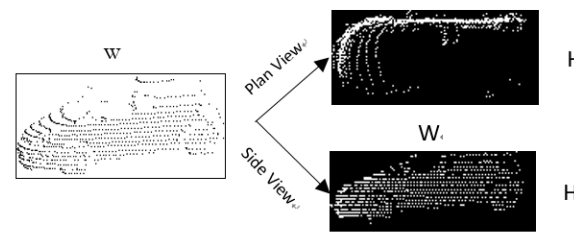

Figure 6. Conversion from 3D points to two 2D images.

Model construction: each point cloud cluster obtained from the roadside laser scanner represents a section of the vehicle. If these sections are patched together according to the correct relationship between every successive overlapped image pair, an aggregated vehicle model will be constructed. Based on the above assumption, the first image is taken as the reference image, then the other images are transformed into the same coordinate system so as to build the model, as illustrated in the model construction step in Figure 5 and Figure 7. There will be a comparison between the model and the target vehicle in terms of the size to assess the accuracy.
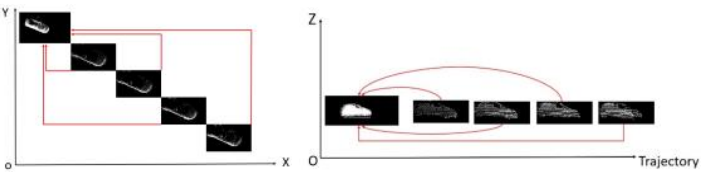

Figure 7. Model construction from plan and side views.

Image registration is performed using template matching, which determines the location of a template within a reference image, to compute the transformation parameters $T_{N, 1}$ between the $N_{\text {th }}$ image and the reference image, as defined in equations (2) and (3).The template image shifts over every possible location in the reference image, pixel by pixel. Considering practical considerations that the vehicle may change its orientation when changing lane or turning, at every shifted location, the template image is rotated from -10 to +10 degrees in increments of 1 degree and a similarity value is calculated for each rotation. The largest value is regarded as the optimum similarity value for this location. The optimum matched position of the template image is the location where the highest similarity value is found and the corresponding rotation is considered as the best rotation. The degree of similarity between the two images, namely the score value as shown in equation (1) (Ding et al., 2001), is calculated with the cross-correlation coefficient metric. A score map can be formed after completion of the searching process (Figure 8).The optimum match is shown as the red dot in the map.

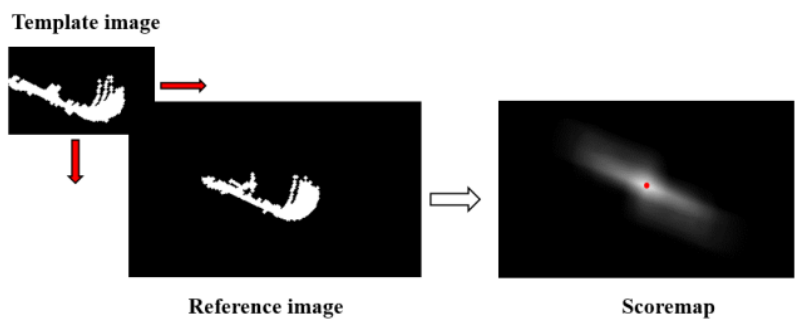

Figure 8 . The matching process.

$$
\begin{gathered}
S_{\theta}(x, y)=\frac{\sum_{i=1}^{m_{1}} \sum_{j=1}^{n_{1}} t_{\theta}(i, j) r(x+i-1, y+j-1)}{\left[\sum_{i=1}^{m_{1}} \sum_{j=1}^{n_{1}} t_{\theta}(i, j)\right]^{1 / 2}\left[\sum_{i=1}^{m_{1}} \sum_{j=1}^{n_{1}} r(x+i-1, y+j-1)\right]^{1 / 2}} \\
P_{i, i-1}=\underset{x, y, \theta}{\arg \max }\left(S_{\theta}(x, y), \theta \in(-10,10)\right) \\
T_{N, 1}=\sum_{i=2}^{N}\left(P_{i, i-1}+\left(X_{i}, Y_{i}, \theta_{i}\right)\right)
\end{gathered}
$$

In equation (1), $t_{\theta}$ is the template with a size of $m_{l} \times n_{1}$ pixels and a rotation of $\theta . r$ is the reference image with a size of $m_{2} \mathrm{x}$ $n_{2}$ pixels. $(\mathrm{x}, \mathrm{y})$ is the origin of the sub-image in the reference image corresponding to the template. In equation (2), $P_{i, i-1}$ is the optimum position of the $i_{t h}$ image in its reference image, namely the $(i-1)_{t h}$ image. In equation (3), $\left(X_{i}, Y_{i}\right)$ is the origin of the $i_{t h}$ image in its enlarged image (in the matching process for each 
image pair, the reference image is enlarged by padding 0 around it in order to guarantee that all the pixels can be searched by the template). $\theta_{i}$ is the rotated angle when the $i_{t h}$ image reaches the optimum position.

Tracking point location from model matching: the centroid of the obtained vehicle model (shown as a red dot in Figure 5), is adopted as the tracking point. Due to the fact that the built model is aggregated by each image containing a certain part of the vehicle according to the transformation parameter $T_{N, l}$, the relative position between the tracked point and each vehicle part in the reference image is to be matched. The tracking point can be back located to the corresponding image if every single image is fitted with the model through a secondary matching. Further conversion back into the original lidar frame can be achieved as an inverse operation of the conversion step.

The main difference in side view tracking compared to plan view tracking is that in the conversion step the vehicle clusters are converted into side images along the vehicle trajectory. As the target vehicle may change its heading direction during the period of observation, to ensure precise tracking, the trajectory orientation should be calculated frame by frame with the variant $\theta$ determined in the plan view tracking for each image. If the orientation of the first vehicle cluster is determined as $\theta_{0}$, the orientation of the $i_{t h}$ vehicle cluster can be inferred by adding the corresponding rotation angle.

The orientation of the first cluster should equal that of the model because the model is built on the first image. To determine its orientation, edges are extracted using the Canny operator (Ding and Goshtasby, 2001) on the model and then lines are determined using the Hough transform (Mukhopadhyay and Chaudhuri, 2015). The direction of the longest line can be regarded as the orientation of the first cluster and therefore the model (Figure 9).
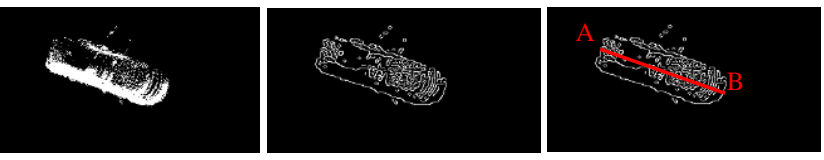

Figure 9. The vehicle model and its orientation.

\section{EXPERIMENTS AND ANALYSIS}

\subsection{Dataset and study area for vehicle detection}

This research employed a RS-LiDAR-32, a cost-efficient $360^{\circ}$ lidar sensor from RoboSense for analysis. The sensor has a detection radius of 0.2 to $200 \mathrm{~m}$ and is designed for various applications, such as autonomous vehicles, robotics, and 3D mapping. It has 32 laser beams, collects data at a speed of $640,000 \mathrm{pts} / \mathrm{s}$ with a scanning frequency of $20 \mathrm{~Hz}$, and covers a $360^{\circ}$ horizontal field of view and a $40^{\circ}$ vertical field of view with $+15^{\circ}$ up and $-25^{\circ}$ downward look angles.

The study area was Claremont Road, located beside the Cassie Building on the campus of Newcastle University, Newcastle upon Tyne, UK (Figure 10). A $360^{\circ}$ camera was installed next to the lidar sensor to provide ground truth for vehicle detection validation.
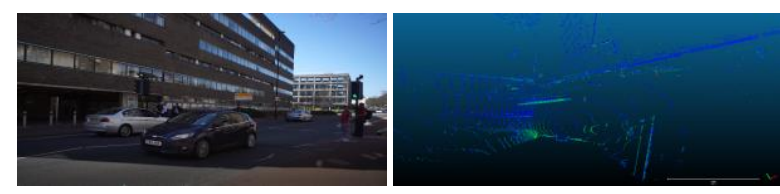

Figure 10. Study area for vehicle detection.

\subsection{Case studies for vehicle tracking}

Two tests were conducted with a vehicle to evaluate the effectiveness of the proposed tracking algorithm. In the acquired dataset there are 550 samples, comprising 375 vehicle samples and 175 non-vehicle samples. The detection performance is evaluated by three indicators: Recall (Rec), Precision $(\mathrm{Pre})$ and F1 score $(2 \mathrm{Rec} \times \mathrm{Pre} /(\mathrm{Rec}+\mathrm{Pre}))($ Table 1$)$.

\begin{tabular}{|c|c|c|c|c|c|c|}
\hline TP & FP & TN & FN & Pre & Rec & $\begin{array}{c}F_{1} \\
\text { score }\end{array}$ \\
\hline 362 & 26 & 12 & 23 & 93.30 & 94.03 & 93.66 \\
\hline
\end{tabular}

Table 1. Vehicle detection accuracy (TP: True Positives; FP: False Positives; TN: True Negatives; FN: False Negatives.).

From two sets of results of the frame-by-frame strategy, shown in Figure 11 and Table 2, the following observations can be obtained:

(1) The RMS values between the tracked velocities from two tracking threads are smaller than $0.25 \mathrm{~m} / \mathrm{s}(0.220 \mathrm{~m} / \mathrm{s}$ for case one, $0.249 \mathrm{~m} / \mathrm{s}$ for case two), which means the displacement deviations are smaller than $0.013 \mathrm{~m}$ during each time interval $(0.05 \mathrm{~s})$. Considering that the image resolution is $0.03 \mathrm{~m}$ (described above), these deviations are within one pixel. As the accuracy of the tracking results can't be better than the adopted pixel-by-pixel matching process, the tracking results from the two views can be regarded as consistent. This consistency demonstrates the reliability of the tracking strategy because the two tracking threads are executed separately.

(2) In case one, the target vehicle accelerated rapidly from a standstill to its maximum speed, then it slowed down slowly to a stop. In case two, the target vehicle entered the scanning area at a constant speed after which it decelerated slightly before speeding up. From Figure 11, it can be seen that the estimated trend of the vehicles are in accordance with real conditions described above, providing further validation for the applicability of the proposed method.

(3) It can be found by further examination of Figure 11 that some large fluctuations occur in plan view tracking; e.g. from $1.25 \mathrm{~s}$ to $1.75 \mathrm{~s}$ (frame 25 to 35 ) in case one and from $2.15 \mathrm{~s}$ to $2.65 \mathrm{~s}$ (fame 43 to 53) in case two. Possible explanations for the fluctuations are as follows: in these frames, only the near edge of the vehicle was scanned, therefore, there was possibly insufficient detail in the corresponding binary plan view images, causing inaccurate matching positions and jumps in calculated velocities. The influence of these fluctuations on the final results is lessened by the counterbalance from side view tracking which is not affected. This analysis demonstrates an advantage of tracking from two views. 


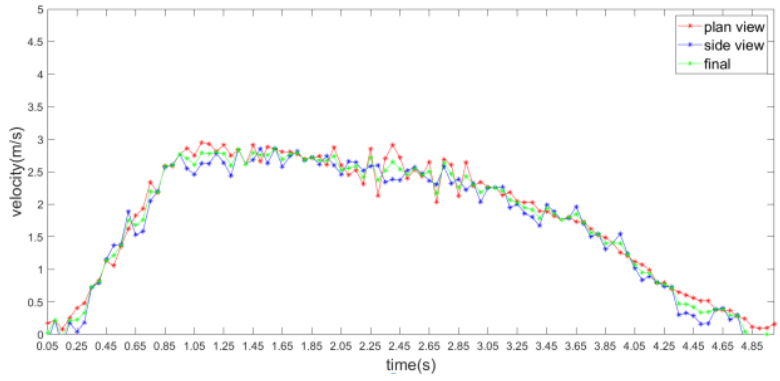

(a) Tracking results for case study one.

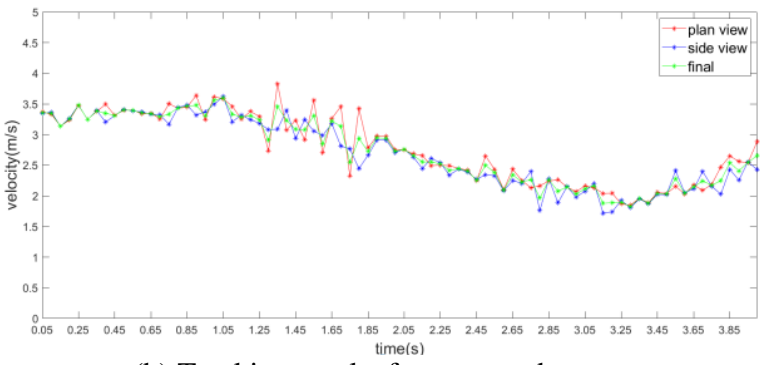

(b) Tracking results for case study two.

Figure 11. Tracking results for the two case studies.

\begin{tabular}{|c|c|c|c|}
\hline $\begin{array}{c}\text { Case } \\
\text { study }\end{array}$ & $\begin{array}{c}\text { Number of } \\
\text { frames }\end{array}$ & $\begin{array}{c}\text { RMS error } \\
(\mathrm{m} / \mathrm{s})\end{array}$ & $\begin{array}{c}\text { Computation } \\
\text { time }(\mathrm{s})\end{array}$ \\
\hline 1 & 100 & 0.220 & 36.5 \\
\hline 2 & 80 & 0.249 & 29.3 \\
\hline
\end{tabular}

Table 2. Parameters for the two case studies.

However, the approach adopted in is study can only perform near field tracking: the average speeds of the two cases are $1.749 \mathrm{~m} / \mathrm{s}$ and $2.736 \mathrm{~m} / \mathrm{s}$, and the tracking time is $5 \mathrm{~s}$ and $4 \mathrm{~s}$, so the tracking ranges are $8.75 \mathrm{~m}$ and $10.95 \mathrm{~m}$, respectively. There are two primary reasons for the short tracking range: firstly, vehicles in the far field do not have a clear shape so that they are removed as false alarms at the detection stage; secondly, occlusions occur when the target vehicle is in the lane furthest from the sensor, greatly influencing the tracking procedure as tracking will cease if the search result is empty in two successive frames during cluster tracking stage. Further work therefore needs to be undertaken to improve robustness and extend the tracking range.

\subsection{Comparison between different methods}

(1) Point and cluster tracking

To better illustrate the advantage of point tracking over cluster tracking, comparative results are shown in Figure 12. The use of the average point of the cluster (which is variable in roadside laser scanning) as the tracking point clearly causes greater fluctuations than the point based approach.

\section{(2) Frame-by frame and model-based tracking}

Frame-by-frame tracking is straightforward: the positions of the point are found in two consequent frames based on the transformation parameters calculated through matching-based image registration. However, image matching will cause registration errors due to its pixel-by-pixel operation. To minimise these random errors, a model is built after aggregating all the frames on the basis of the calculated transformations. In these two cases, the length offset between the model and the target vehicle is within $30 \mathrm{~cm}$. Taking the image resolution $(0.03 \mathrm{~m})$ and the total number of tracking frames (100 in case one, 80 in case two) into account, the model can be regarded as accurate enough for further usage. The tracking point can then be located to frames after secondary matching them with the model. Figure 13 shows the comparative results between frameby-frame and model-based tracking. Although showing a similar pattern, there is a small offset between the two sets of results. Unfortunately, as insufficient ground truth data exists in the current test dataset, further trials are required to validate the model-based approach.

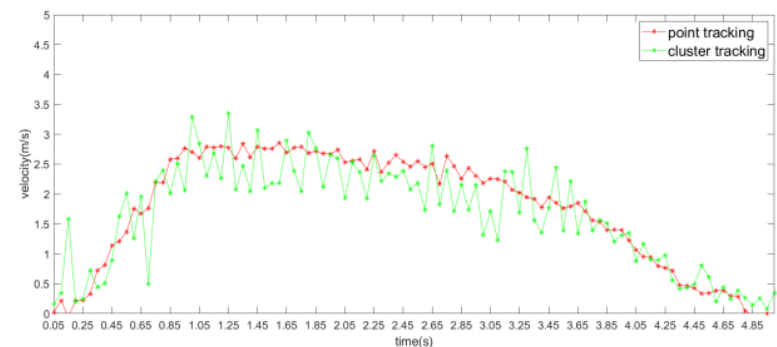

(a)

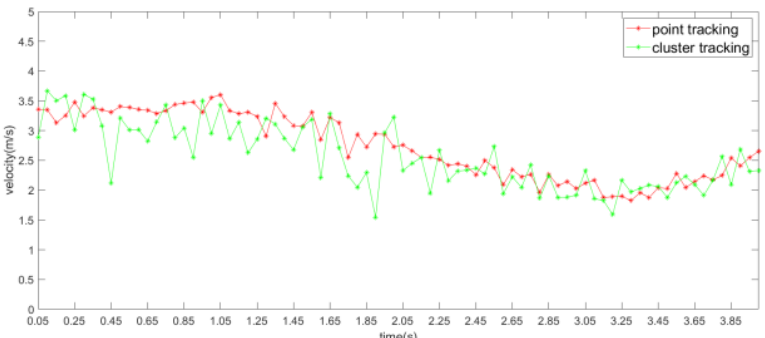

(b)

Figure 12. Comparison between point and cluster tracking results.

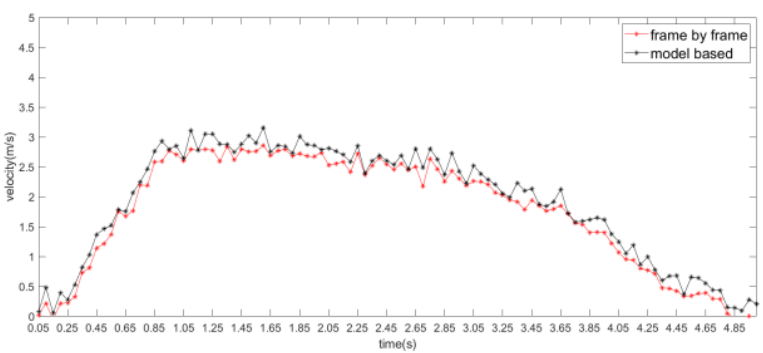

(a)

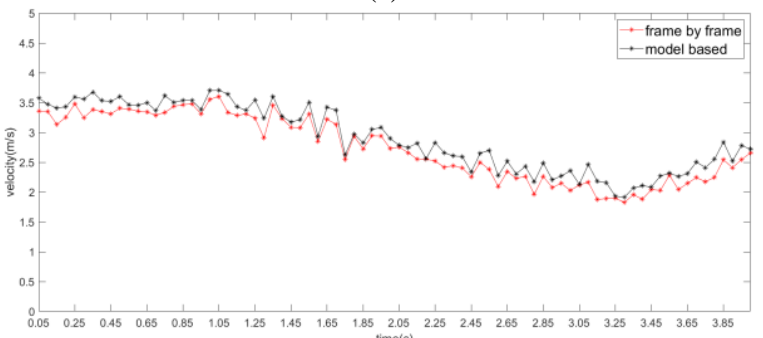

(b)

Figure 13. Comparison of frame-by-frame and model-based tracking results.

\section{CONCLUSIONS}

This paper reports research to develop an image-based vehicle tracking approach from roadside lidar data. The 3D point cloudbased vehicle tracking problem is decomposed into two imagebased vehicle tracking procedures from a plan and side view 
along the tracked vehicle's trajectory. A point tracking operation has been added in addition to traditional cluster tracking to improve tracking precision.

The main contributions of this work are twofold: (1) more precise vehicle tracking is provided via point instead of cluster tracking; (2) the twin-track procedures provide opportunity to validate the results from two different views. The effectiveness of this method has been evaluated through two case studies. Further work will focus on extending the range of the vehicle tracking by applying new algorithms to make the procedures more robust and deploying multiple sensors along the roadside. Moreover, camera imagery can supplement lidar data to achieve better vehicle detection performance.

\section{ACKNOWLEDGEMENTS}

This research was supported by a China Scholarship Council (CSC) Studentship, No. 201706370243 held by Jiaxing Zhang at Newcastle University, UK. The research was also supported by UKCRIC - UK Collaboratorium for Research in Infrastructure \& Cities: Newcastle Laboratories (EPSRC award EP/R010102/1).

\section{REFERENCES}

Asvadi, A., Girão, P., Peixoto, P., Nunes, U., 2016. 3D object tracking using RGB and LIDAR data. In: 19th IEEE International Conference on Intelligent Transportation Systems, 1255-1260.

Besl, P.J., McKay, N.D., 1992. Method for registration of 3-D shapes. IEEE Transactions on Pattern Analysis and Machine Intelligence, 14(2), 239-256.

Byun, S., Jung, K., Im, S., Chang, M., 2017. Registration of 3d scan data using image reprojection. International Journal of Precision Engineering and Manufacturing, 18(9), 1221-1229.

Chen, T., Wang, R., Dai, B., Liu, D., Song, J., 2016. Likelihood-field-model-based dynamic vehicle detection and tracking for self-driving. IEEE Transactions on Intelligent Transportation Systems, 17(11), 3142-3158.

Christodoulou, A., 2018. An image-based method for the pairwise registration of mobile laser scanning point clouds. In: The International Archives of the Photogrammetry, Remote Sensing and Spatial Information Sciences, Vol. XLII-4, 93-100.

Demantké, J., Mallet, C., David, N., Vallet, B., 2011. Dimensionality based scale selection in $3 \mathrm{~d}$ lidar point clouds. In International Archives of the Photogrammetry, Remote Sensing and Spatial Information Sciences, vol. XXXVIII-5/W12, 97102 .

Ding, L., Goshtasby, A., 2001. On the Canny edge detector. Pattern Recognition, 34(3), 721-725.

Ding, L., Goshtasby, A., Satter, M., 2001. Volume image registration by template matching. Image and Vision Computing 19(12), 821-832.

Godin, G., Rioux, M., Baribeau, R., 1994. Three-dimensional registration using range and intensity information. In: Proc. SPIE 2350, Videometrics III.

Held, D., Levinson, J., Thrun, S., 2013. Precision tracking with sparse 3d and dense color 2d data. In: 2013 IEEE International Conference on Robotics and Automation, 1138-1145.

Hwang, S., Kim, N., Choi, Y., Lee, S., Kweon, I.S., 2016. Fast multiple objects detection and tracking fusing color camera and 3D LIDAR for intelligent vehicles. In: 13th IEEE International Conference on Ubiquitous Robots and Ambient Intelligence, 234-239.

Khalfan, A., Li, H., Andrews, G., 2015. Cold Start SI Passenger Car Emissions from Real World Urban Congested Traffic. In: SAE technical paper series. SAE World Congress. Society of Automotive Engineers.

Lin, C.-C., Tai, Y.-C., Lee, J.-J., Chen, Y.-S., 2017. A novel point cloud registration using $2 \mathrm{~d}$ image features. EURASIP Journal on Advances in Signal Processing, 2017(1), 5.

Luo, Z., Habibi, S., Mohrenschildt, M.V., 2016. LiDAR Based Real Time Multiple Vehicle Detection and Tracking. World Academy of Science, Engineering and Technology, International Journal of Computer, Electrical, Automation, Control and Information Engineering, 10(6), 1125-1132.

Mukhopadhyay, P., Chaudhuri, B.B., 2015. A survey of Hough Transform. Pattern Recognition, 48(3), 993-1010.

Sanchez, A., Suarez, P.D., Conci, A., Nunes, E., 2011. Videobased distance traffic analysis: application to vehicle tracking and counting. Computing in Science \& Engineering, 13(3), 3845 .

Sanchez, J., Denis, F., Checchin, P., Dupont, F., Trassoudaine, L., 2017. Global Registration of 3D LiDAR Point Clouds Based on Scene Features: Application to Structured Environments. Remote Sensing, 9(10), 1014.

Shirazi, M.S., Morris, B.T., 2017. Looking at intersections: a survey of intersection monitoring, behavior and safety analysis of recent studies. IEEE Transactions on Intelligent Transportation Systems, 18(1), 4-24.

Sun, Y., Xu, H., Wu, J., Zheng, J., Dietrich, K.M., 2018. 3-D data processing to extract vehicle trajectories from roadside LiDAR data. Transportation research record, 2672(45), 14-22.

$\mathrm{Wu}$, J., 2018. An automatic procedure for vehicle tracking with a roadside LiDAR sensor. Institute of Transportation Engineers. ITE Journal, 88(11), 32-37.

Xiao, W., Vallet, B., Schindler, K., Paparoditis, N., 2016. Simultaneous detection and tracking of pedestrian from panoramic laser scanning data. ISPRS Annals of Photogrammetry, Remote Sensing and Spatial Information Sciences, Vol. III-3, 295-302.

Xiao, W., Vallet, B., Schindler, K., Paparoditis, N., 2016. Street-side vehicle detection, classification and change detection using mobile laser scanning data. ISPRS Journal of Photogrammetry and Remote Sensing, 114, 166-178.

Xiao, W., Vallet, B., Xiao, Y., Mills, J., Paparoditis, N., 2017. Occupancy modelling for moving object detection from lidar point clouds: A comparative study. ISPRS Annals of Photogrammetry, Remote Sensing \& Spatial Information Sciences, Vol. IV-2/W4, 171-178.

Yao, W., Zhang, M., Hinz, S., Stilla, U., 2012. Airborne traffic monitoring in large areas using LiDAR data-theory and experiments. International journal of remote sensing, 33(12), 3930-3945. 